\title{
Estimating the Economic Value of Flexibility in Access Network Unbundling
}

\author{
Koen Casier, Mathieu Tahon, Mohsan Ahmed Bilal, Sofie Verbrugge, \\ Didier Colle, Mario Pickavet, and Piet Demeester
}

\author{
Ghent University - IBBT - IBCN, Gaston Crommenlaan 8, 9050 Gent, Belgium \\ \{koen.casier, mathieu.tahon, sofie.verbrugge, \\ didier.colle, mario.pickavet, piet.demeester\}aintec.ugent. be
}

\begin{abstract}
By means of local loop unbundling (LLU), the incumbent operator opens the access network connecting the customer to the central office to other operators. Other licensed operators (OLO) will use this possibility to provide customers their services. The price of the LLU-offer is set by a regulator, aiming to allow fair competition in a monopolistic market. This is typically fixed at the lowest possible price covering all of the incumbent's costs in this network. Now, the OLO always has the choice between the regulated LLUoffer, own installation or not providing access to the customer. In order to remove any unfair competition advantages, the regulator should incorporate a flexibility bonus in its LLU-pricing strategy. In this paper we show how the economic technique of real option valuation can be used for estimating a numeric value for this flexibility advantage.
\end{abstract}

Keywords: Local Loop Unbundling, Techno-Economic, Real Options, Telecom Regulator.

\section{Origin, Context and Impact of Local Loop Unbundling}

In Europe a lot of the telecom networks have evolved out of community or countrywide installed copper networks. Considering the importance of those telephone networks, this company was placed under the control of the government, which gave additional stimuli to grow this network country-wide, connecting every inhabitant. While the networks were originally installed for providing telephony signal transmission, later on they also allowed for data transmission. With the advent of ADSL, the importance of digital data transmission over this network grew. Soon data took over as the driving factor for network upgrades. Clearly at the other side, the network connected to a very diverse set of services. At this point in time, the publicly owned telecommunication network keeps off all competition and innovation in this field.

A large privatization wave decoupled most of the so-called incumbent network operators in Europe from direct control of the government. Still the existing network infrastructure, which had been fully installed and paid for in the public era, poses a large advantage to the use of these incumbent operators. Without clearing this unfair 
historic advantage, new entrants will never have a chance to compete in the former monopolistic market. To counter this negative effect on competition, the national telecom regulators have imposed the incumbent network operators the obligation to open up their existing infrastructure to the new entrants often called other licensed operators (OLO). When considering the access network, this obligation is called the local loop unbundling (LLU) and the regulator will fix the price based on an estimation of the maintenance costs of this network for the incumbent. In extension the operator might also be forced to open up at the data link layer which is often called bitstream access. Opening the access network at any possible place to the OLOs is referred to as LLU in the remainder of this paper.

In this study we considered the case of a copper based network operator who is faced with a regulatory fixed price for giving the OLOs access to his network. As mentioned this price is based on an estimation of the costs for maintenance (and other costs) of this selected part of the network for the incumbent. It is considered a fair price covering the costs the incumbent indulges. We get a better picture of the full interaction and economic impact when looking from the point of view of the OLO. The OLO can choose, at each point in time and at each location, to connect customers. In this choice he will invest a small amount on the equipment to be collocated in the central office (CO). For each customer connected he will pay the fixed price directly covering the costs of the incumbent, and as such the OLO will have a competitive case against the incumbent for each customer. On top of that, the OLO has a higher flexibility as he can choose where and when to start connecting and how many customers to connect. He can also pull out from certain regions when they are no longer profitable and shift equipment and attention to other locations. When used optimally this higher flexibility allows the OLO to maximize revenues, minimize costs and risks, and have a more profitable overall business case than the incumbent.

The economic value of such flexibility is less straightforward to estimate in the business case and is as such often neglected. In order to judge on the impact of this flexibility, one should use more advanced calculations. In this paper we show how we used the theory of real options to calculate the economic value of the flexibility, in the remainder of the paper referred to as options, of the OLO. This economic evaluation technique has been used abundantly in the past to estimate the impact of uncertainty and managerial flexibility on the outcome of a business case [1], [2], etc. In [3] and [4] the same issue of estimating the value of LLU for an OLO through the use of real options is addressed. In our paper we go one step further, providing a quantitative full-fledged study combined with realistic figures for the calculation of the OLOs advantage.

In section 2 we build a full quantitative model for the calculation of the costs for a copper based OLO. We also indicate what unbundling offers this operator can use in the construction of his own business case. In order to reflect the economic impact of the choices the OLO has at hand, in section 3 this business case is enhanced with real options. We also indicate which uncertain factors will have the highest impact on the case and on the choices to be taken. The outcome is an extended business case for the OLO in which he will always choose the optimal installation path given the uncertain input. Confronting this model with the original business case gives an idea of the value of the options in LLU. The second part of this section shows several realistic 
results. Finally section 4 aggregates all information available in the paper and distils some general and more detailed conclusions from this.

\section{Forecasting the Costs for an OLO}

Access to existing network infrastructure can be opened up at different levels. Theoretically the network can be opened at each level of functionality, for instance as defined in the OSI layered model. In the context of a copper based network, the network is typically unbundled in the following ways, numbered according to the OSI layer at which they are opened:

0. Full Unbundling (FU): The incumbent patches the copper pair running to a customer directly to the equipment of the OLO. This requires the equipment of the OLO to be placed in collocation with the incumbent's installation in the CO. In this approach, the OLO has full access to the physical medium at OSI layer 0.

1. Shared Line (SL): The incoming copper pair is led through a splitter, where voice is separated from data. Both signals are brought to either incumbent or OLO. At this point, the physical (OSI) layer 1 is fully defined for both voice (PSTN) and data (xDSL).

2. Bitstream access (BA): The incumbent splits voice from data in the CO. The data is sent further into the metro network and is transported to a fewer number of aggregation switches. At these aggregation switches (called point of presence or POP), the OLO can collocate his equipment in order to reach the customer. The connection from this point up to the customer is handled on OSI layer 2 or 3.

Figure 1 gives an overview of these three possibilities for opening up the network.

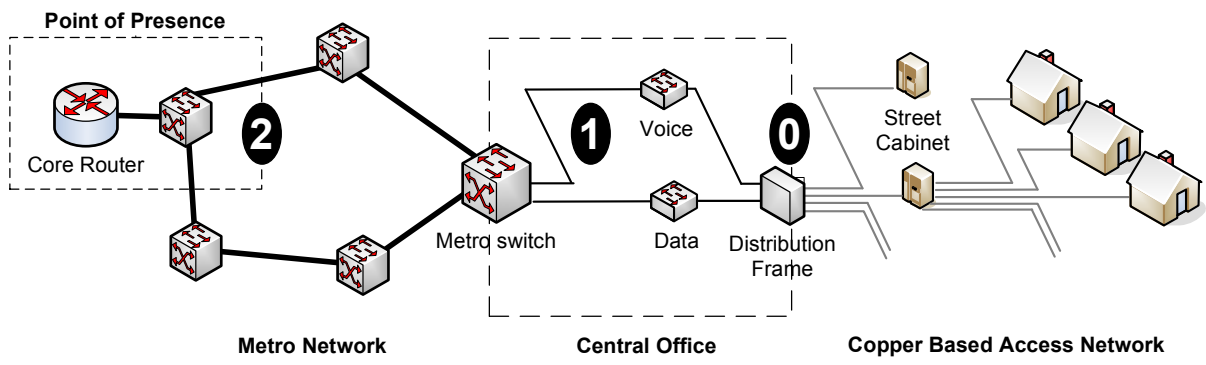

Fig. 1. Overview of the network from access (right) to core (left) with an indication of the unbundling locations

\subsection{Forecasting the Market}

In general the OLO takes his share of the total telecom market. Still the incumbent operator often holds a major part of the market and leaves only a small margin for the different OLOs to capture. In the context of this research we considered the Belgian 
market, in essence a duopoly in which the cable and copper operator are in fierce competition. We focus on the copper network segment, in which Belgacom is the incumbent and is regulated nationally by the Belgian regulator BIPT. The OLO market in Belgium represents a share somewhere below $15 \%$ or less than $500 \mathrm{k}$ residential connections [5]. The same source also contains an overview of the occurrences of the different types of LLU used by the OLOs. For estimating the market for the OLO, we started from the growth of broadband in Belgium, modeled using a Gompertz curve (sales indicated by $\mathrm{S}(\mathrm{t})$ ) (1) fitted to the values found in [5] (resulting in $[m, a, b]=[3.6 \mathrm{M}, 5.25,0.32]$ ). Within this overall customer potential, we modeled a market segment for all OLOs in the market, initially set at $15 \%$. In following years, this market segment has an uncertainty of growth based on the previous year. We limited the growth to between $-5 \%$ and $5 \%$ of the customer base in the market segment.

$$
\begin{array}{rll}
S(t)=m \cdot e^{-e^{-b \cdot(t-a)} \text { with }} \quad m & \text { market potential } \\
a & \text { infliction point (37\% adoption) } \\
b & \text { slope of the adoption }
\end{array}
$$

\subsection{Installation of Equipment}

In the given situation the market should be split in the smallest individual regions in which the OLO can compete with the incumbent. As mentioned before the CO location is the first place at which the OLO can connect customers (FU or SL). In each of those individual markets, the OLO can select its strategy and build a smaller economic model. In order to connect customers here, the OLO will install copper connected data and/or voice equipment in the $\mathrm{CO}$ of the incumbent. In addition, the OLO will also have to pay a LLU rental fee per actual customer to the incumbent. Additionally the OLO will also have a growth in its overall operations as a result of the new installation in this region. On the positive side, the OLO will also gain income from the customers connected in the region.

In case the OLO would rather prefer to connect part of the customers using BA, he will place data equipment at a higher aggregation point in the POP. Again he will have to pay an LLU rental fee to the incumbent on a per customer base.

\subsection{The Overall Cost Model}

The overall cost model is obtained by combining the different building blocks and is shown in Figure 2. The background of how to build the techno-economic model and how to calculate process and infrastructure costs can be found in [6]. A very important parameter in this context is the cost of goods sold (COGS) which is actually the tariff the OLO will have to pay to the incumbent for using the LLU offer for connecting the customers. We used this model on an installation set of $500 \mathrm{COs}$ (FU and SL) and one aggregation POP (BA) at which the OLO can connect. In [5] and [7] the proportion of FU, SL and BA are known and we spread this proportion randomly over the $500 \mathrm{COs}$. Additionally we have randomized the customer base for each $\mathrm{CO}$ according to a logarithmic cumulative function in which 35 COs connect $50 \%$ of the customers and 100 COs connect $70 \%$ of the customers. The combination of the costs 
and revenues in all COs with collocated equipment (FU or SL) and the results for the different POPs and associated customers gives the final cashflow of the business case for each year. Summing these cashflows, while taking into account the time value of money, gives the net present value (NPV) of the OLOs business case. We considered a time span of 5 years for the evaluation of the business case. Of course the selection whether to use BA or FU/SL can be optimized for each region and in each year. This flexibility will be dealt with in the following chapter.

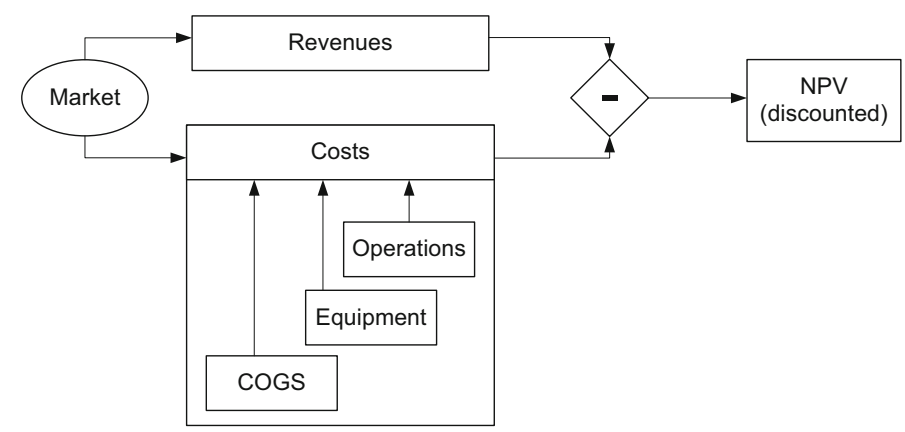

Fig. 2. Overall cost model used for calculating the outcome for the OLO

Although this case has been constructed in the context of the Belgian situation, it did not use all real input values and can as such not be translated to reality without considerable changes in the input values. The conclusions expressed in this paper are not statements explicitly valid for Belgium but only valid for our theoretical model with its specific hypothesis and cost elements.

\section{Introducing Flexibility and Estimating Its Value}

The NPV calculation presented above cannot capture flexibility. Once a project is set out, the project path is fixed. However, most real business cases have built-in flexibility. The management can at every point of time decide to follow a different path, e.g. abandoning the project after one year when the payoff does not meet the requirements. The economic theory proposed an extension of the NPV model to implement flexibility, the real option approach. The real option valuation methodology tries to capture (and include) the value of managerial flexibility present in a business case, much in the same way the flexibility presented in financial options (over stocks) is valued. A financial option gives the right to buy or sell over a limited period the underlying asset for a predetermined exercise price. As it is a right (and not obligation) the value of an option will always be positive. The technique of Real Options was defined in 1977 [8] and applies option pricing theory to the valuation of investments in real assets. It proved especially useful in investment decisions consisting of different (optional) phases. As it adds flexibility to the business case, it alleviates (partly) the estimation of the risk by means of the discount factor as in the calculation of the net present value. 
The value of a project should now be extended by the value of the options, and is defined as the summation of the original NPV of the project, as calculated in Section 2 , with the value of each of the options. Several option valuation techniques are in use in economic literature. Black and Scholes and Binomial tree valuation (and extensions) are used in theoretical real option studies. These valuation techniques are generally not feasible for larger studies in which several options are modeled and several uncertainties are taken into account. In this case a Monte Carlo simulation is used on the model taking into account all options and uncertainties. The model is simulated 10000 times, every time generating a NPV. The result of such a simulation is a distribution of all results. The outcome mean NPV of this model will already reflect the summation of original NPV and option values. [9] provides a more extensive introduction to Real Options theory, with a lot of practical examples.

\subsection{Integrating Real Options with the Techno Economic Model}

In the setup of the cost model, we already indicated some of the most important uncertainties for an OLO. We incorporated some additional uncertainties into the real option evaluation of the business case and indicate below their average value and standard deviation. We also indicate additional information on the expected probability distribution (assumptions) where necessary (in general we assumed a normal distribution).

- $\quad$ Average Return per User (ARPU)

Average ARPU of $€ 30$ per month per user with a standard deviation of $€ 1$. A higher ARPU will of course lead to a more positive business for the OLO.

- Churn rate

Average Churn of $15 \%$ with a standard deviation of $1.5 \%$.

- Regulatory context

Binary chance (50/50) for a change in the regulation (LLU pricing) in the coming years. Consecutive changes have been inversely correlated in order to reduce instability with year on year changes in regulations.

- $\quad$ Equipment pricing

We estimated a price erosion on the equipment, diminishing the costs yearly. To reflect the uncertainty on equipment pricing, the yearly calculated cost is changed additionally using a multiplier. This multiplier is modeled by a normal distribution on average $100 \%$ and with a standard deviation of $2 \%$. (cheaper or more costly)

- $\quad$ OLO Market

Both the full OLO market and the position for one OLO within this market are uncertain. The full OLO market is expected to follow the $15 \%$ of the total broadband adoption. On top of this we expect both the full market and the position for one OLO in this market to change using a multiplier. This multiplier is modeled by a normal distribution on average $100 \%$ and with a standard deviation of $2 \%$.

In real options we try to estimate the effect of managerial flexibility to counter the negative effects and gain from the positive, considering the given uncertainties. This flexibility is incorporated in the model by means of options, choices that can be made 
and will alter the outcome of the business case. Here the OLO always has the option to select the type of LLU for each separate area - CO in case of FU or SL, or all CO's connected to the same POP in case of BA - separately. In order to keep the calculations feasible, we restricted the options in two ways. First we did not expect an OLO to switch from $\mathrm{CO}$ collocation to BA for a given area. This is a reasonable assumption as switching back would involve a lot of upfront investments (COequipment) to go up in smoke. Once the CO-equipment is installed, FU or SL will also almost always be the cheaper option. Secondly we also restricted the different options to be taken at the end of each year instead of continuously executable options. Figure 3 gives a visual representation of the remaining options.
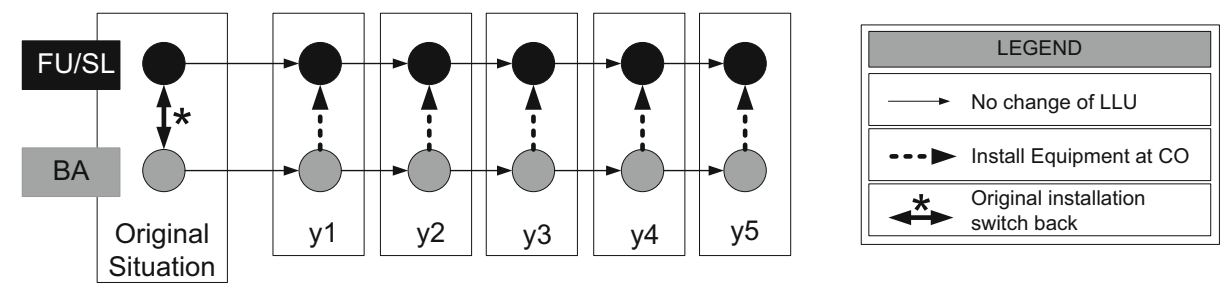

Fig. 3. A visual overview of the connection options for the OLO at the end of each year

Considering the complex nature of the cost estimation model and the large amount of uncertain factors influencing the outcome of this case, we choose to calculate the value of the different options in the business case using a Monte Carlo simulation based approach. Of course we calculated the outcome in the case of a full optimal managerial decision in each case. Additionally, we calculated the outcome in case the OLO would (FU) only be able to provide access over FU or SL, (BA) only be able to provide BA, (No) be stuck with its existing installation, (Thresh.) start from the existing situation and switch using a threshold of 480 users per $\mathrm{CO}$. To be complete we refer to the full optimal use of the options as (Opt.). In this latter case, the operator can also make use of a free correction in the first year (as if he is a new OLO).

\subsection{Results of Real Options}

One of the main parameters in the business case is the position of the considered OLO within the full OLO market. Figure 4 gives a view on the outcome of the business case for an OLO with a market share of $10 \%$ up to $100 \%$.

The first thing to notice is how an OLO with less than $30 \%$ of our expected $(15 \%)$ OLO market, meaning $4 \%-5 \%$ of the total Broadband Market will have real difficulties in reaching a positive result. Once the OLO has more than $30 \%$ of the OLO market, he has a good chance of reaching a positive business case and its margins are increasing for an increasing market share.

Next the results also clearly show how the full optimal strategy has a good advantage over the non-flexible situation (either FU, BA or No) and Figure 55 shows a value of the options in a full optimal situation. We find an option value of $€ 9$ per customer for an OLO with a positive business case (40\% market share) when comparing to FU. This value is rapidly decreasing to between $€ 2$ and $€ 1$ for an 


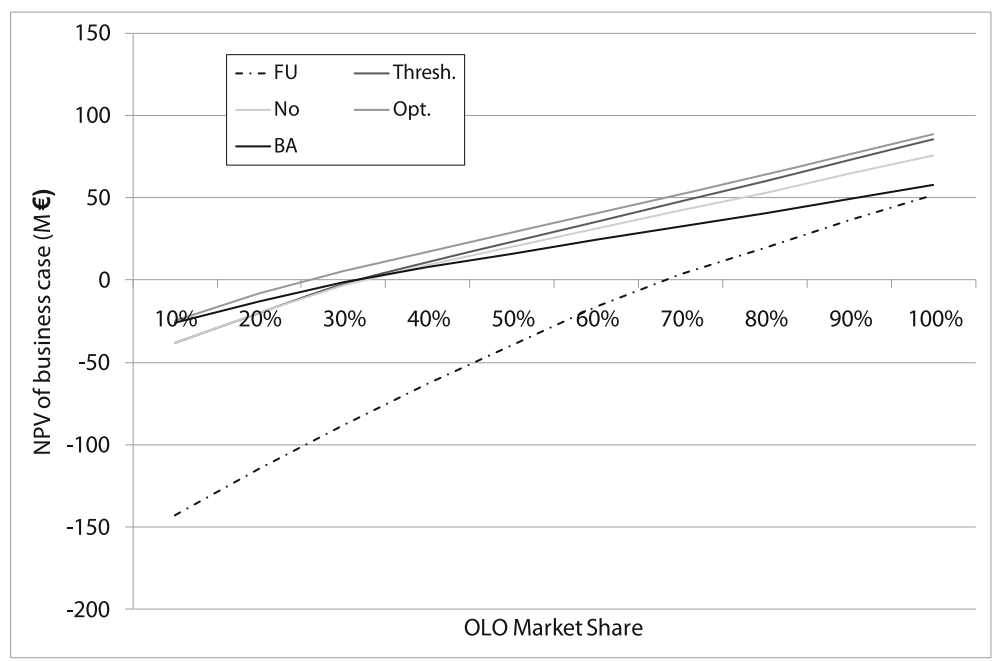

Fig. 4. NPV of the business case for an OLO using the different options

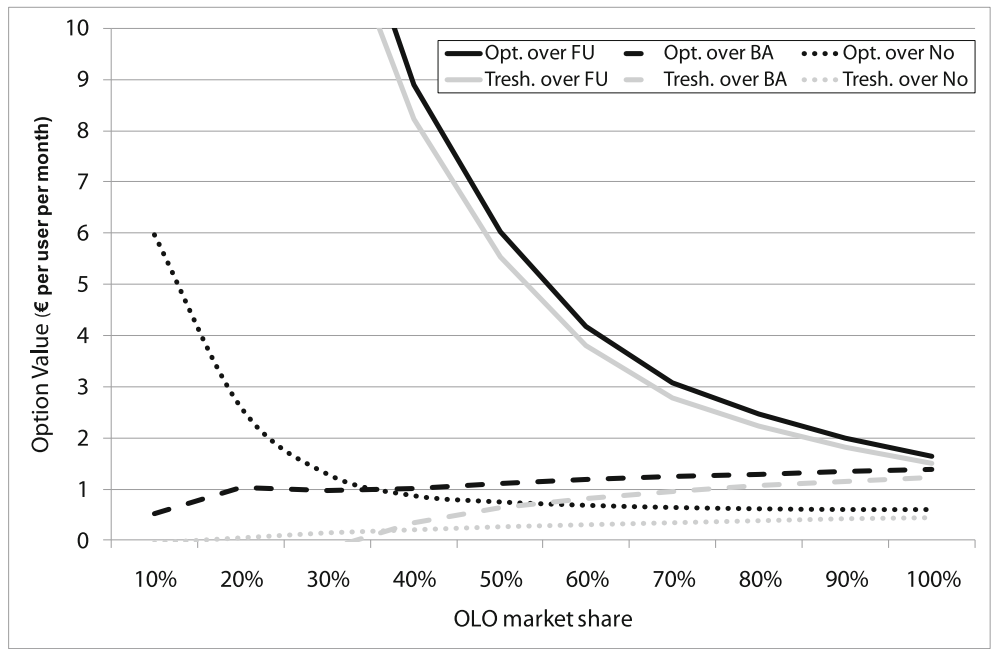

Fig. 5. The value of the different options at the OLO's disposal

increasing market share. When comparing to BA we find an almost flat option value of $€ 1$ between $40 \%$ and full market share.

Basing the option of switching on a predefined threshold is only slightly worse and will still give a good option value over a CO-based installation. However for a market share of less than $30 \%$ the value of the option becomes negative. As the estimation of the managerial response is based on some upfront decision, rather than the selection of the real optimum, this calculation is strictly speaking not a real option valuation. Still this simulation of a likely managerial response in a threshold based rule gives 
valuable information. It requires less knowledge and outlook to make the decision and still incorporates some intelligence towards the decision (at least above a market share of $40 \%$ ). In this context it is important to note that the threshold based selection will start from the existing situation and can as such not switch back in the first year (as is allowed for Opt. and is assumed in BA. Allowing this based on the predefined threshold, for which the results are shown in Figure 66, makes the rule more generally applicable and gives a positive (average) option value in all considered cases.

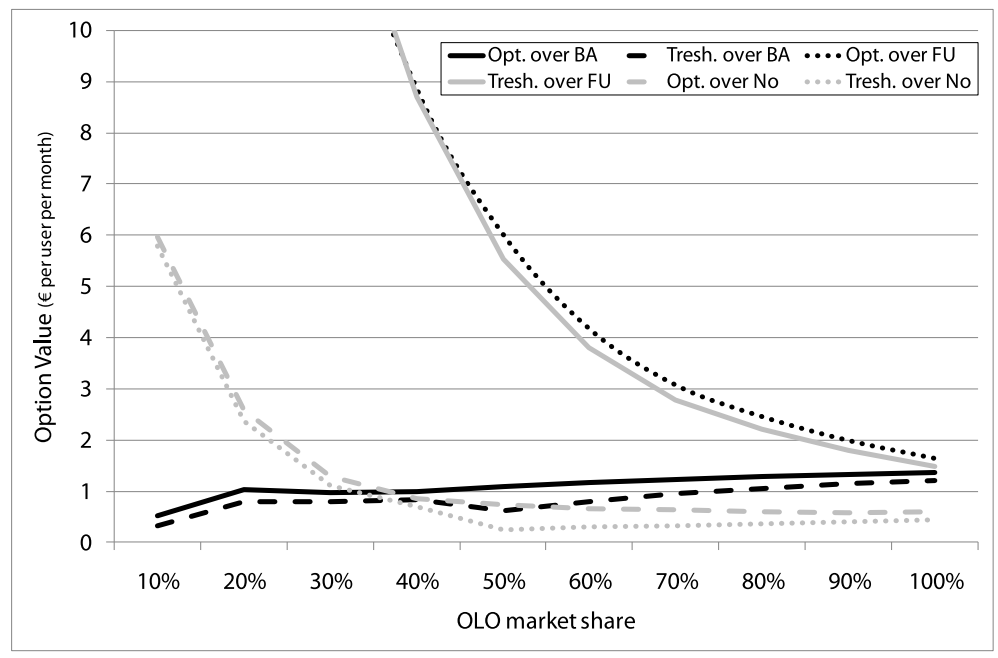

Fig. 6. The value of the different options with improved threshold based option

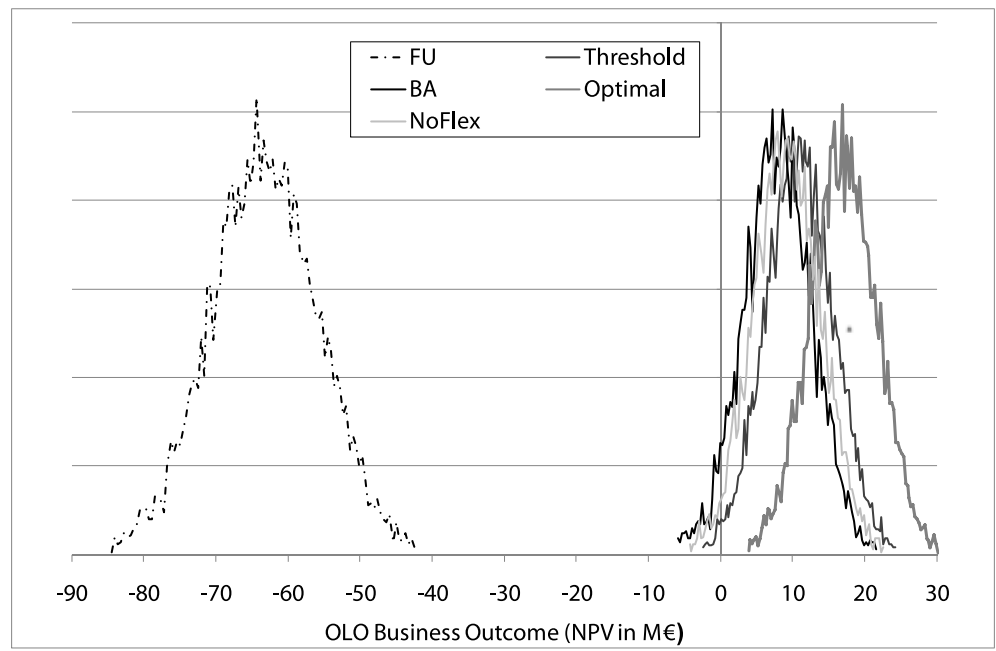

Fig. 7. NPV distribution for an OLO with $40 \%$ market share 
As mentioned before, the business case becomes (on average) positive for an OLO on condition he has a market share at least $40 \%$ of the OLO market ( $6 \%$ of the total market) in most strategies. The outcome NPV in this case is shown in Figure 7 for all different strategies for a variation in input as used in the Monte Carlo simulation. Clearly chances of a negative business case are very low in case at least BA is provided to the OLO. In case both LLU offers are provided, a much higher result is attainable. The highest result is logically found by selecting the highest result in each case as shown in Opt..The sensitivity analysis performed in this case also gives important information on the most important uncertainty parameters for the simulations. Market share is without any question the most important.

\section{Conclusions}

In many European countries, incumbent telephony operators had a monopoly on the telecom market. Historically they were rolled out under monopoly on a country-wide scale. The copper infrastructure has already been fully installed and paid for the in the past and this network provides the incumbent with a intangible advantage on the market. By means of local loop unbundling (LLU), the regulator forces the incumbent operator to allow other licensed operators (OLO) to connect customers over the existing network. The OLO will have to pay the incumbent on a per customer base for this usage, reflecting the incumbents costs over this network. In such a regulated market, the OLO has a high flexibility of choosing where to connect which customers. Traditional investment analysis techniques cannot take this flexibility into account and are not useable for measuring the value of this flexibility for the OLO. Still this value is very important. In the first place, this additional flexibility undermines the fair market competition for the incumbent player and when no counter measures are taken it gives no incentives to the OLOs to rollout new own equipment. A solution to this problem is the use of real options to incorporate the value of flexibility and managerial options into the business case.

We have constructed a techno-economic model for estimating the costs of an OLO for providing connections to its customers on top of an existing copper based network. We used the real option valuation approach in the paper and were able to calculate the option value for the OLO. For the hypothetical case we estimated an option value up to $€ 9$ per line per month, a value which can clearly not be neglected. The business case of an OLO already becomes profitable once he reaches $40 \%$ or more of the OLO market. As such, up to two OLOs can perfectly co-exist with the existing incumbent. In case the market has a considerable size, a third OLO focusing on this opportunity could still make a positive business case.

These results clearly show that the value of this flexibility cannot be neglected and should be taken into account by a regulator when fixing the prices and options for LLU. Setting the tariff too high will of course scare the OLOs from the market. Setting the tariff too low on the other hand, might break down future competition as it gives no incentive to follow the ladder of investment for an OLO and might squeeze the margins of the incumbent and kill all incentives there for upgrading his network or rolling out his own infrastructure. 
The current approach considered a copper based network, but the question of LLU stays as important for new network installations. A valuable extension to this study would be the combination of both to estimate the flexibility of LLU in a new fiber network. Also mobile networks for which we already constructed some economic models [10], [11] could be extended with this line of reasoning. Even beyond the field of telecommunications, other infrastructures such as electricity, gas and water which were recently privatized in Europe, exhibit similar possibilities for opening access to the transport network. Finally the case considered in this paper only mentions one incumbent player, whereas in a duopoly installation, future networks or international connections, more than one player could be offering LLU over the same (or overlapping) region. In this case OLOs could gain even more from the additional flexibility they have for switching operators.

\section{References}

1. Camacho, F.T., Menezes, F.M.: Access Pricing and Investment: A Real Options Approach. Competition and Regulation in Network Industries CRNI, JEL L51 (2008)

2. Sadowski, B.M., Verheijen, M., Nucciarelli, A.: From Experimentation to Citywide Rollout: Real Options for a Municipal WiMax Network in the Netherlands, Communications \& strategies, No. 70, 2nd Q (2008)

3. Pindyck, R.: Mandatory Unbundling and Irreversible Investment in Telecom Networks. NBER Working Paper No. 10287, JEL No. L0 (2004),

http: / /web.mit.edu/rpindyck/www/Papers / Mandatory_Unbundling.pdf

4. Haussman, J.A.: Regulated Costs and Prices in Telecommunications. In: The handbook of Telecommunications Economics, Edward Elgar, vol. II (2000)

5. Organisation for Economic Co-operation and Development, Broadband statistics, http: / / www. oecd.org/

6. Verbrugge, S., Casier, K., Van Ooteghem, J., Lannoo, B.: Practical steps in technoeconomic evaluation of network deployment planning. In: IEEE Global Communications Conference, Exhibition and Industry Forum (Globecom 2009), Honolulu, Hawaii, USA, November 30-December 4 (2009)

7. Nationaal Instituut voor de Statistiek, http: //www. statbel. fgov. be

8. Myers, S.: Determinants of Corporate Borrowing. Journal of Financial Economics 5(2), 147-175 (1997)

9. De Maeseneire, W.: The real options approach to strategic capital budgeting and company valuation, Financiële Cahiers, Larcier (2006) (ISBN: 2-8044-2318-2)

10. Van Ooteghem, J., Verbrugge, S., Casier, K., Pickavet, M., Demeester, P.: Future proof strategies towards fiber to the home. In: ICT-Public \& Private Interplay Next Generation Communication conference in Sevilla, December 10-12 (2008)

11. Van Ooteghem, J., Verbrugge, S., Casier, K., Lannoo, B., Pickavet, M., Demeester, P.: Competition and Interplay Models for Rollout and Operation of Fiber to the Home Networks. Working paper (2009) 